\title{
Alternative ground cover for lettuce production under tropical climate conditions
}

Ernandes Guedes Moura ${ }^{1}$

Fábio de Sousa Silva²

Rodrigo Fonseca da Silva ${ }^{3}$

Fabrício Ribeiro Andrade ${ }^{4}$

Jodean Alves da Silva 5

Ana Teresa Pereira da Silva ${ }^{6}$

\section{Abstract}

Alternative farming techniques to increase lettuce quality and productivity, rising environmental and social development, have been the subject of several studies. The objective of this study was to select economic and readily available cover materials to enhance the production of curly lettuce, South of Piauí. It was used a randomized block design with four replications, with the following ground cover treatments: rice husk (Oryza sativa L.), chopped andropogon grass straw (Andropogon gayanus Kunth), sawdust powder and control (without ground cover). The andropogon grass straw can be recommended for lettuce production under the prevailing edaphoclimatic conditions in Uruçuí city, located Southwest of Piauí state.

Keywords: cv. Grand Rapidis. Mulching. Lettuce production. Organic waste.

\section{Introduction}

Lettuce (Lactuca sativa L.) stands out for being the most consumed leafy vegetable in Brazil (SALA; COSTA, 2012) and it is of great economic value (QUEIROGA et al., 2002; GRANGEIRO et al., 2006). These authors also claim lettuce production is low Northeast of Brazil, compared to areas with mild temperatures, which hindrances its growth and development, preventing the expression of its genetic potential, as well as leaving the domestic demand unattended.

Lettuce can be produced in climates under high temperature and low precipitation like southwest of Piauí state, year-round (SOUSA et al., 2013), nevertheless these weather conditions can lead to bolting and leaf malformation (FILGUEIRA, 2008) which makes cultivation practices necessary in order to minimize these effects.

1 Federal Institute of Education, Science and Technology of Maranhão, São João dos Patos (MA), Brazil. ernandes.guedes@ifpi.edu.br 2 Federal Institute of Education, Science and Technology of Piauí, Uruçuí (PI), Brazil. fabiosousasilva01@ifpi.edu.br 3 Federal University of Piauí (UFPI), Agronomic Engineering Department (DEN), rodrigo11.07@hotmail.com. CEP: 64900-000, Bom Jesus (PI), Brazil. (author for correspondence)

4 Federal Institute of Education, Science and Technology of Mato Grosso (IFMT), Juína (MT), Brazil. fabricio.andrade@jna.ifmt.edu.br.

5 Federal University of Lavras (UFLA), Department of Water Resources, Lavras (MG), Brazil. jodean2013@gmail.com.

6 State University of Piauí (UESPI), Graduate in Agronomy, Uruçuí (PI), Brazil. anateresa@hotmail.com. 
Agricultural techniques that increase productivity and crop quality (OLIVEIRA et al., 2010; BLAT et al., 2011; SILVA et al., 2011; DALRI et al., 2014; PINTO et al., 2016) with rational use of resources has been increasingly demanded; considering the efficiency of the use of nutrients varies accordingly to climatic conditions, soil type, irrigation and management systems, among other factors, it is necessary investing more in technology, reducing costs and improving product quality in a sustainable manner (NASCIMENTO, 2012).

Ground cover has multiple functions: increasing water infiltration, reducing the impact of rain and erosion, maintaining a constant temperature and soil moisture, enriching the soil with nutrients after decomposition while decreasing weeds emergence and reducing nutrients leaching, which improve crop performance (ARAÚJO et al., 1993; SOUZA; RESENDE, 2006; RODRIGUES et al., 2009; FERREIRA et al., 2014; SILVA et al., 2015). Several materials are used as soil cover, highlighting plastic mulching, coffee shells, double-sided low density plastic film, grass, banana leaves, sawdust, black agrotextil, rice husks, among others (REGHIN et al., 2002; STAL; DUSKY, 2003; ANDRADE JUNIOR et al., 2005; RODRIGUES et al., 2009; BRANCO et al., 2010; BRANDÃO et al., 2016).

Avoiding application of industrial soil cover materials reduces production costs (ARAÚJO NETO et al., 2009). Furthermore, managing organic residues in the soil improves its physicochemical properties, contributing to a high quantitative and qualitative yield of lettuce (YURI et al., 2004; GALVÃO et al., 2013). However, producers rely on available and viable techniques; therefore, handling other types of coverage sets a priority for the region (YURI et al., 2004; FERREIRA et al., 2014).

Studying the effect of different materials (rice straw and coffee husk, Brachiaria, sawdust and control without cover) in lettuce productivity, Carvalho et al. (2005) concluded the best results were obtained using mulching organic material on the site regardless of the type of surface cover.

The southwest region of Piauí state is characterized by an average daily temperature of $27{ }^{\circ} \mathrm{C}$ throughout the year, although it is possible to reach temperatures close to $40{ }^{\circ} \mathrm{C}$ (VIANA et al., 2002). Consequently, producers are compelled to search inexpensive and efficient techniques that provide better quality by improving the appearance of products in the competitive market. To achieve such objectives, this study aims to select low-cost and readily accessible soil cover materials to increase the production of curly lettuce, South of Piauí State.

\section{Material and methods}

The experiment was conducted under field conditions March/April 2014, at the horticultural sector of Federal Institute of Education, Science and Technology (IFPI), Campus Uruçuí, located in the PI-247, at the Cerrado Portal neighborhood in the city of Uruçuí (PI) (Latitude 070 14' 02" S; Longitude 44 33' 14" W) (FIGURE 1), which belongs to the Southwestern of Piauí state, mesoregion and to Alto Parnaíba microregion. The climate, according to Köppen classification, is Aw, hot and semi-humid. The average annual rainfall is $1.000 \mathrm{~mm}$ and the mean temperature is $29^{\circ} \mathrm{C}$ (SOUSA et al., 2013). 
Figure 1. Location of the study area, at Federal Institute of Education, Science and Technology of Piauí (IFPI), Campus Uruçuí (PI), Brazil.

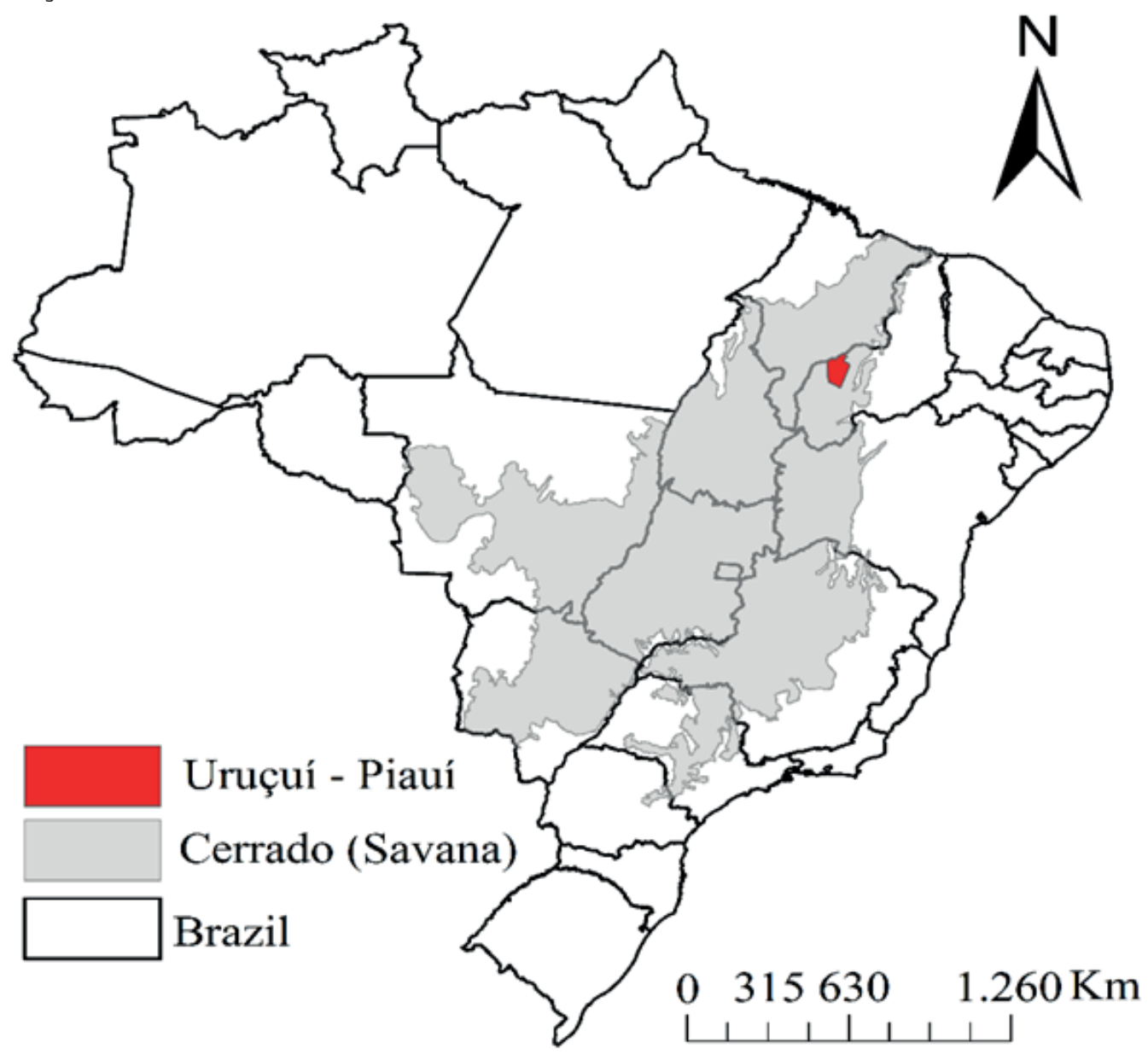

Source: Prepared by the authors (2016).

The cultivar used in this study was the Grand Rapids curly type, widely cultivated among local producers, which gained commercial acceptance, showing high productivity. The seeds were sown in polystyrene trays containing 128 cells, the organic substrate base, plant soil and carbonized rice straw in the ratio of 2:1:1 proportion. As soon as at least three leaves have already developed, 20 day old seedlings were transplanted to $1.00 \mathrm{~m}$ wide by $2.00 \mathrm{~m}$ long seedbeds under field conditions, whose soil characteristics are shown on Table 1.

Table 1. Chemical characterization of the soil in the study area.

\begin{tabular}{|c|c|c|c|c|c|c|c|c|c|}
\hline $\mathrm{pH}$ & $P$ & K & $\mathrm{H}+\mathrm{Al}^{3+}$ & $\mathrm{Al}^{3+}$ & $\mathrm{Ca}^{2+}$ & $\mathrm{Mg}^{2+}$ & SB & CEC & $\mathrm{OC}$ \\
\hline $\mathrm{H}_{2} \mathrm{O}$ & \multicolumn{2}{|c|}{---mg dm ${ }^{-3}---$} & \multicolumn{6}{|c|}{ 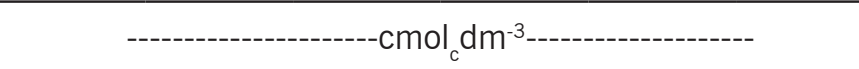 } & $-\%-$ \\
\hline 5.30 & 2.30 & 31.00 & 3.30 & 0.10 & 0.90 & 0.70 & 1.68 & 4.98 & 1.90 \\
\hline
\end{tabular}

$\mathrm{P}$ and $\mathrm{K}$ extracted with Mehlich ${ }^{-1} ; \mathrm{Ca}, \mathrm{Mg}$ and $\mathrm{Al}$ extracted with $\mathrm{KCl} ; \mathrm{H}+\mathrm{Al}$ extracted with SMP buffer; $\mathrm{CEC}-$ cation exchange capacity; $\mathrm{OC}$ - organic carbon.

Source: Prepared by the authors (2016). 
A standard fertilization with bovine manure (20 $\mathrm{Mg} \mathrm{ha}^{-1}$ ) was performed at all sites. In addition, a supplemental mineral fertilizer containing 15,55 and $30 \mathrm{~g}$ was added per plot of nitrogen ( $N$ ), $\mathrm{P}_{2} \mathrm{O}_{5}$ and $\mathrm{K}_{2} \mathrm{O}$, respectively. Urea was used in the amount of $18 \mathrm{~g}$ per plot as $\mathrm{N}$ cover fertilization 10 days after transplantation.

The experimental layout was a randomized block design with four replications, and the following types of ground cover (treatments) were adopted: rice husk (CA), chopped andropogon grass straw (Andropogon gayanus Kunth) (PCA), sawdust powder (PS) and the control (TEST).

Organic material was added in two centimeter layer thickness, 10 days after transplantation in the plots. Each plot had four rows per bed, with a total of 24 plants, spaced $0.30 \mathrm{~m}$ between them, resulting in $2.16 \mathrm{~m}^{2}$ per plot. There were eight central plants of each plot, occupying an area of $0.72 \mathrm{~m}^{2}$.

Daily irrigations were made to restore water loss through evapotranspiration, maintaining soil field capacity. Hoeing for weed control was performed 10 and 20 days after transplantation. There was no need to apply pesticides. Plants were harvested 40 days after transplantation, production characteristics and quality of the plants were evaluated.

Plant height was measured from soil surface to the first apical leaves. After the plants harvesting, the thickness of the stem below the first leaves was determined using a digital caliper. The number of leaves was measured from the basal leaves to the last fully expanded leaf. The average production of fresh phytomass (expressed in g) from eight plants was obtained from the center of the seedbeds, using a $0.001 \mathrm{~g}$, semiquantitative balance.

The analysis of variance (ANOVA) was performed once the assumptions were verified (FERREIRA et al., 2012). Comparison of means for the sources of variation and their interactions was performed by Tukey test at $5 \%$ probability, using the R software version 3.2.5 (R CORE TEAM, 2016).

\section{Results and discussion}

The analysis of variance revealed a significant influence $(p<0.05)$ of different types of ground cover, on the crop growth in the edaphoclimatic conditions in Uruçuí city. Positive mulching effects observed on plants development (SOUZA; RESENDE, 2003) are mostly a reflection of a biologically more suitable environment (FIALHO et al., 1991), low water loss and less soil temperature fluctuations (BRAGAGNOLO; MIELNICZUK, 1990), especially in the first few centimeters, which favors greater surface roots proliferation (CARTER; JOHNSON, 1988).

Plant height exhibited variability between the different soil cover (FIGURE 2) which is corroborated by the results obtained by Mógon and Câmara (2007) and provided better performance when compared to those observed by Reghin et al. (2002). Lettuce reached a height of $26 \mathrm{~cm}$ when andropogon grass was added, a higher value than those obtained by Reghin et al. (2002) and Cavalcante (2008), who observed a growth of 20 and $21 \mathrm{~cm}$, respectively. This difference may be due to culture sensitivity to adverse temperature conditions, factor crucial for the growth rate, which may change the plant development cycle, therefore changing the harvest period. 
Figure 2. Plant height ( $\mathrm{CV}=27.07 \%$ ) of lettuce $\mathrm{cv}$. Grand Rapidis as a function of different soil covers. Means followed by the same letter do not differ by Tukey test at $5 \%$ probability. TEST $=$ control; CA = rice husk; PS

= sawdust powder and PCA = andropogon grass straw.

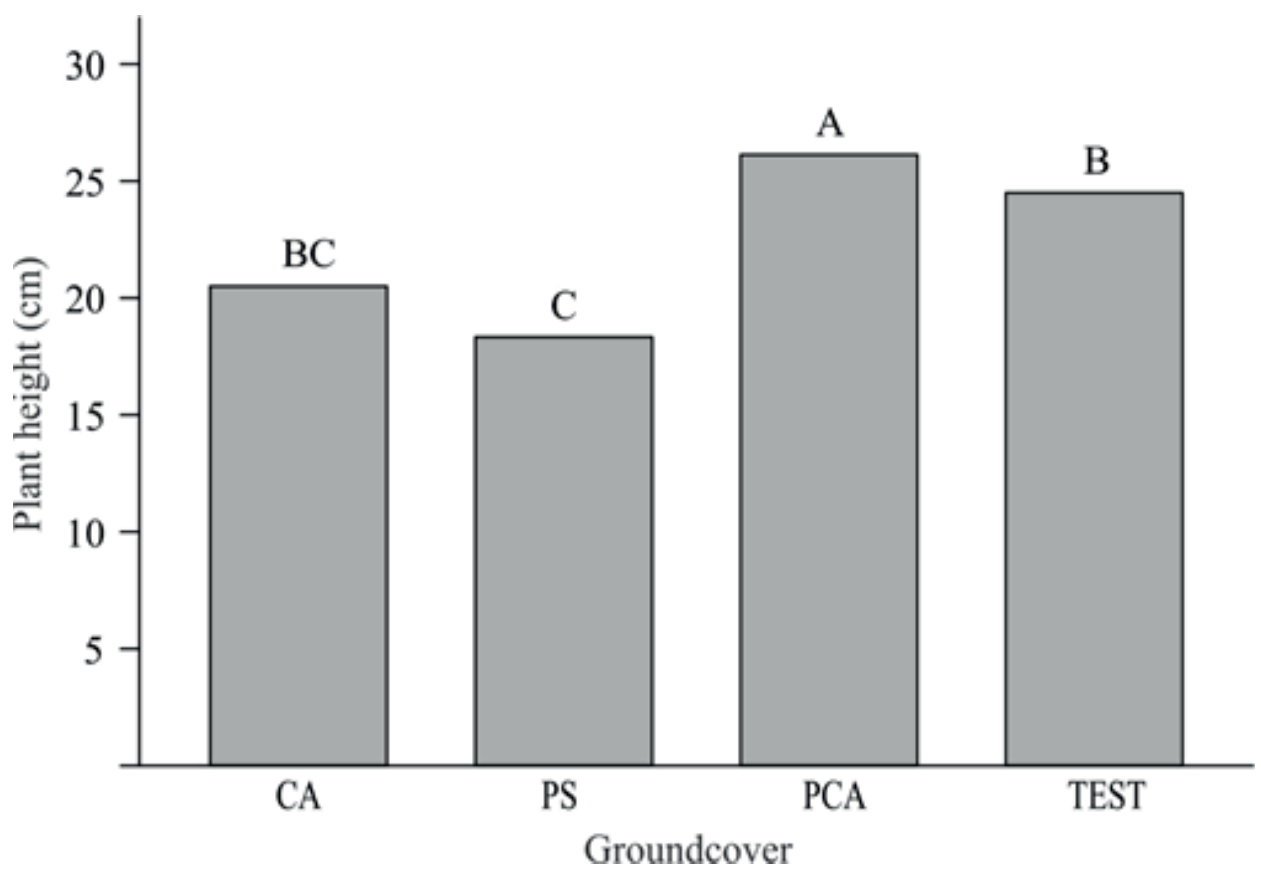

Source: Prepared by the authors (2016).

The lowest lettuce yield was obtained from sawdust powder treatment and may be related to the quality of cover material. Burés (1997) reports that factors such as type of wood, storage time and tannin content can influence the quality of this subproduct, resulting in anaerobic fermentation during the aging processes and generating organic acids which interfere with the growth of roots, a main limiting factor.

The andropogon grass straw promoted the highest values for stem diameter, but it did not differ from rice husk and the latter did not show differences from the control (FIGURE 3). A similar trend was observed by Andrade Junior et al. (2005), who found an average stem diameter of 20.3 and $21.6 \mathrm{~mm}$, respectively, for Regina and Elisa cultivars, in Três Corações, south of Minas Gerais state, Brazil. The stem diameter, an important component in this variety of lettuce, supports the leaves and these edible parts should not be in contact with the ground, to avoid infestation diseases, whilst preserving the quality and quantity of the product. Highest diameter values were obtained from the mulching treatments and such results are linked to a favorable microclimate in the soil, in as much as reducing the evaporation of water on the surface thereof decreasing soil temperature fluctuations, optimal conditions for nutrients absorption (ANDRADE JUNIOR et al., 2005). 
Figure 3. Stem diameter (CV $=13.40 \%$ ) of lettuce $\mathrm{CV}$. Grand Rapidis as a function of different soil covers. Means followed by the same letter do not differ by Tukey test at $5 \%$ probability. TEST = control; CA $=$ rice husk; PS = sawdust powder and PCA = andropogon grass straw.

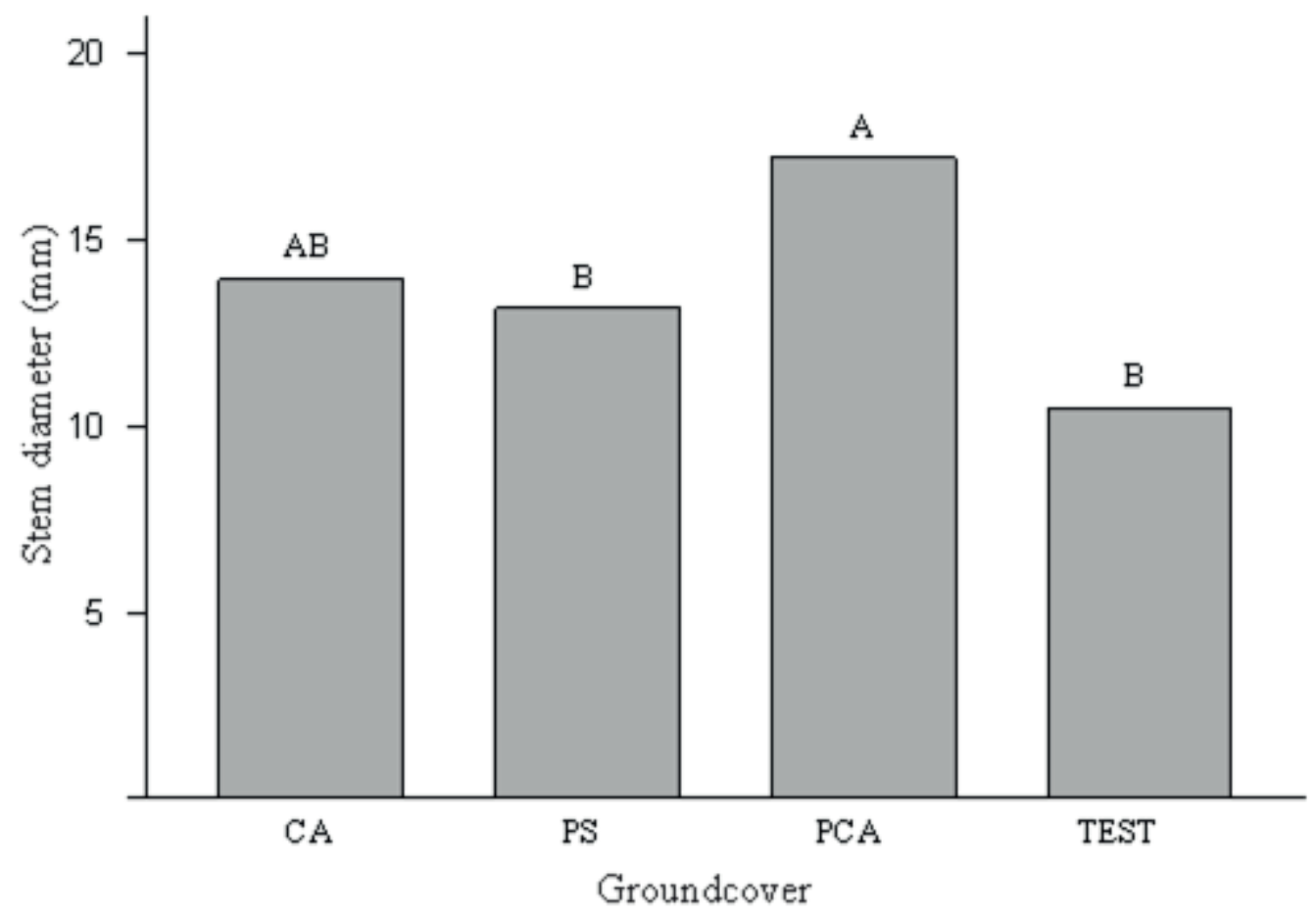

Source: Prepared by the authors (2016).

Number of leaves (FIGURE 4) varied depending on the ground cover. Andropogon grass was different from the control, producing around 19 leaves plant ${ }^{-1}$. The addition of organic wastes under the ground surface increased the number of leaves per plant compared to the control (12 leaves plant $^{-1}$ ). The behavior observed when rice husk and sawdust were applied may be related to the process of temporary immobilization of nutrients, particularly $\mathrm{N}$, required in the process of decomposition performed by soil microorganisms (CARRIJO et al., 2004). The decomposition process was faster in chopped andropogon grass straw due to the lower C:N ratio. Accordingly, Oliveira et al. (2009) found organic compounds increased microbial biomass because of a higher carbon to nitrogen ratio, which may favor the development of lettuce. Reghin et al. (2002) found there were no statistical differences between the number of leaves per plant (average of 24 leaves), using black agrotextil and rice husks as ground cover compared to bare soil. 
Figure 4. Number of leaves per plant ( $\mathrm{CV}=14.04 \%$ ) of lettuce cv. Grand Rapidis as a function of different soil covers. Means followed by the same letter do not differ by Tukey Test at $5 \%$ probability. TEST $=$ control; CA $=$ rice husk; PS = sawdust powder and PCA = andropogon grass straw.

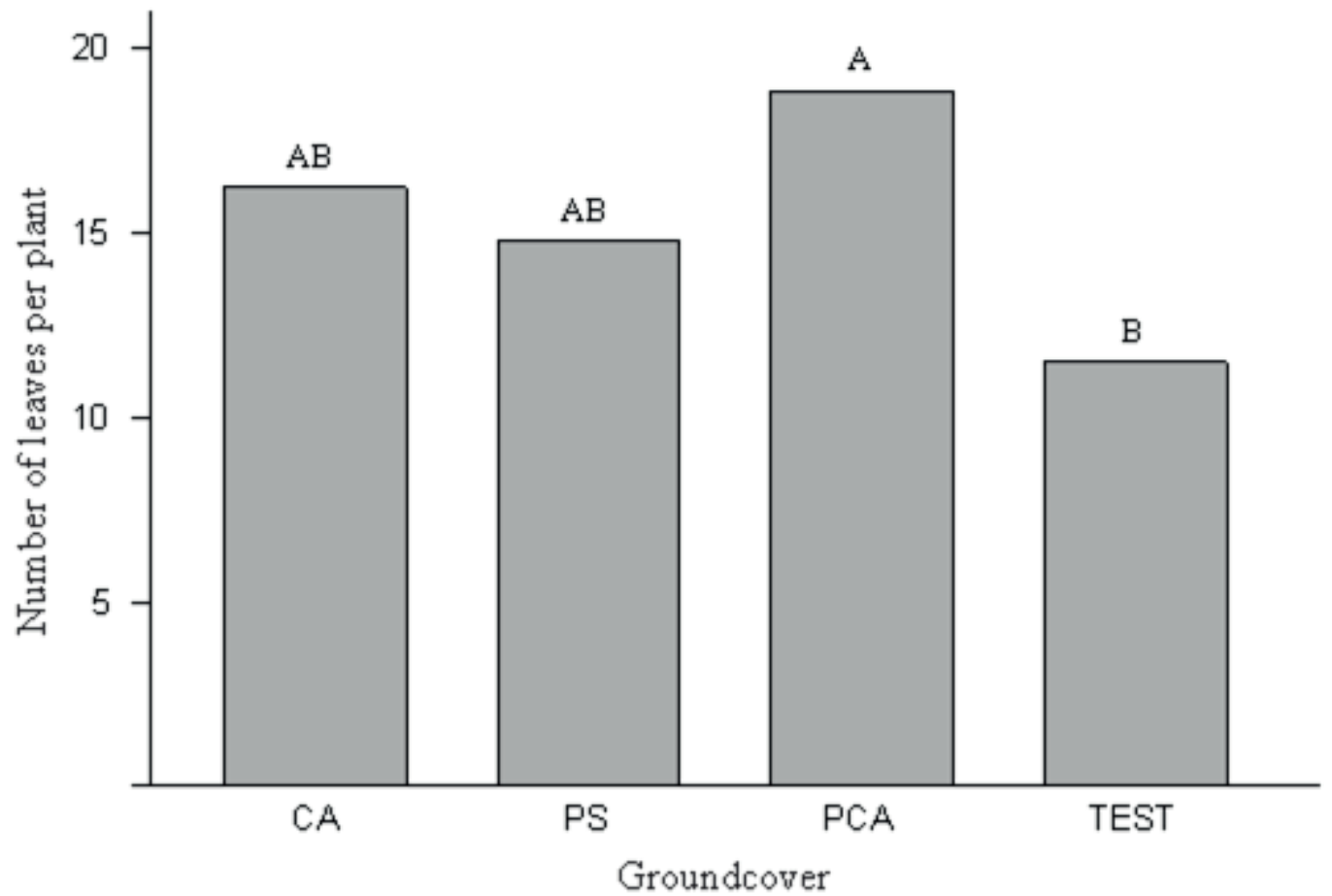

Source: Prepared by the authors (2016).

Chopped andropogon grass had the highest productivity (g. planta $^{-1}$ ), similar to the effects observed for rice husk (FIGURE 5) with no differences between these treatments. The result obtained in this study is similar to Moura Filho (2009) findings, at which mulching showed superior performance in productivity than control (no cover). The lower productivity adopting sawdust may be related to harmful chemical compounds released by this material in its early decomposition, detrimental to lettuce development, as pointed out by Santi et al. (2010).

Thus, the application of alternative organic residues is a strategy to achieve the socioeconomic development of producers in Uruçuí, allowing a cheap and plentiful lettuce production. 
Figure 5. Production ( $C V=22.24 \%$ ) of lettuce $\mathrm{cv}$. Grand Rapidis as a function of different soil covers. Means followed by the same letter do not differ by Tukey Test at $5 \%$ probability. TEST = control; CA = rice husk; PS

$=$ sawdust powder and PCA $=$ andropogon grass straw.

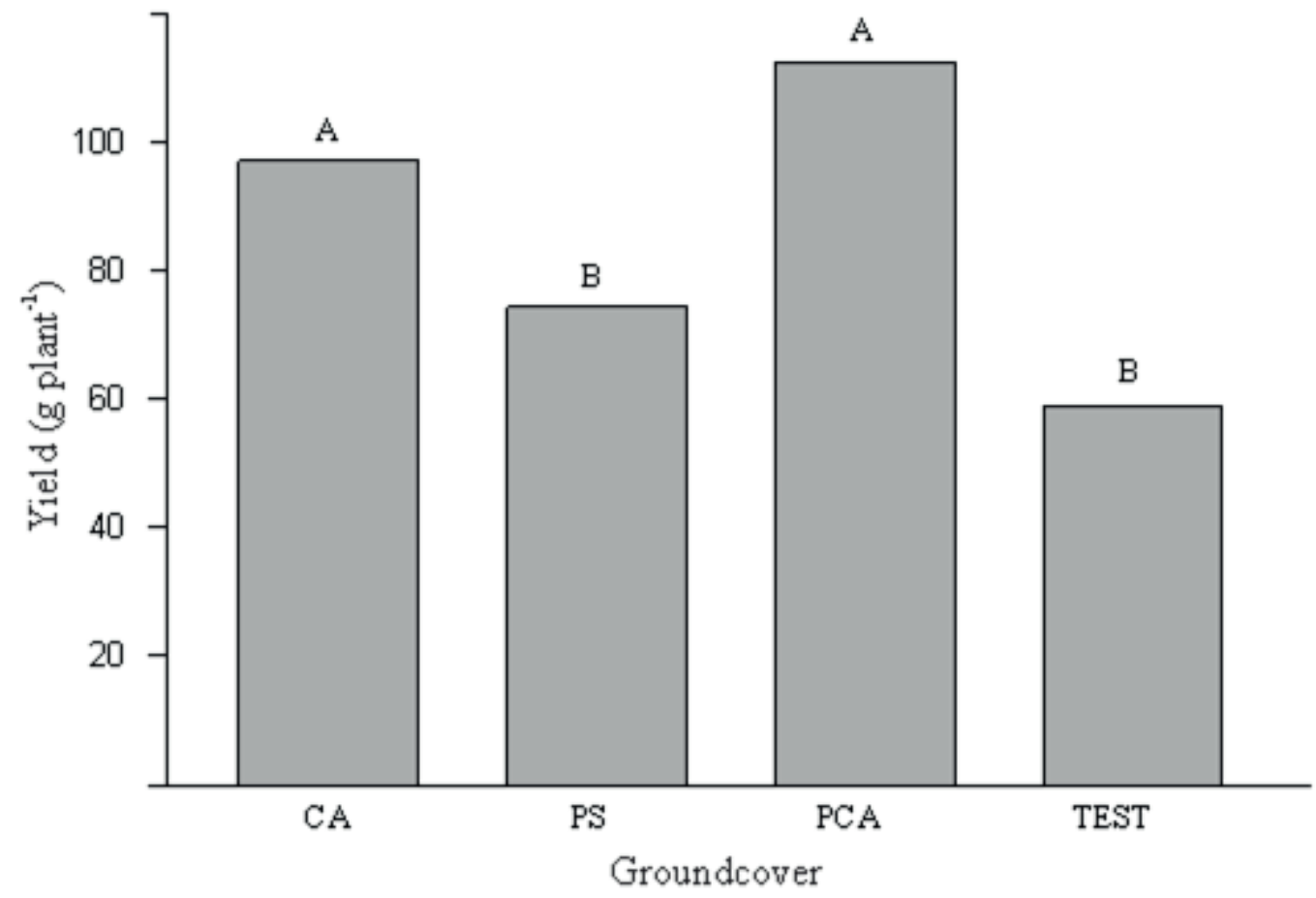

Source: Prepared by the authors (2016).

\section{Conclusions}

1. Lettuce development was positively influenced by alternative groundcover materials.

2. Andropogon grass straw can be recommended for lettuce production at the edaphoclimatic conditions in Uruçuí, Southwest Piauí State.

\section{Acknowledgment}

The authors want to thank Federal Institute of Education, Science and Technology of Piauí (IFPI), which provided the experimental area and the agricultural laboratory facilities for the study. They also acknowledge the funding agencies CAPES and CNPq for granting the scholarship and research funding.

\section{Cobertura alternativa do solo para produção de alface sob condições de clima tropical}

\section{Resumo}

A busca por técnicas agrícolas alternativas, visando a um aumento da produtividade e qualidade da cultura alface, bem como a um melhor desenvolvimento socioambiental, tem sido objeto de 
diversas pesquisas. Neste sentido buscou-se com o presente estudo selecionar materiais de cobertura do solo de fácil aquisição e baixo custo que elevem a produção da alface crespa, nas condições do sul do estado do Piauí. O delineamento experimental utilizado foi em blocos casualizados com quatro repetições, sendo os tratamentos de cobertura do solo: casca de arroz (Oryza sativa L.), palha picada de capim andropogon (Andropogon gayanus Kunth), pó de serra e a testemunha (sem cobertura sobre o solo). O emprego de palha de capim andropogon pode ser recomendado para a produção de alface para as condições edafoclimáticas de Uruçuí no sudoeste piauiense.

Palavras-chave: cv. Grand Rapidis. Cobertura morta. Produção de alface. Resíduos orgânicos.

\section{References}

ANDRADE JÚNIOR, V. C.; YURI, J. E.; NUNES, U. R.; PIMENTA, F. L.; MATOS, C. S. M.; FLORIO, F. C. A.; MADEIRA, D. M. Emprego de tipos de cobertura de canteiro no cultivo da alface. Horticultura Brasileira, Brasília, v. 23, n. 4, p. 899-903, 2005. Disponível em: <http://www.scielo.br/scielo. php?script=sci_arttext\&pid=S0102-05362005000400007> . Acesso em: 23 dez. 2018.

ARAúJO, R. C.; SOUZA, R. J.; SILVA, A. M.; ALVARENGA, M. A. R. Efeitos da cobertura morta do solo sobre a cultura do alho (Allium sativum L.). Ciência e Prática, Lavras, v. 17, n. 3, p. 228-233, 1993. Disponível em: < http://www.bdpa.cnptia.embrapa.br/consulta/busca?b=ad\&id=752482\&biblioteca =vazio\&busca = autoria:\%22ALVARENGA,\%20M.A.\%22\&qFacets=autoria:\%22ALVARENGA,\%20M.A.\%22\&sort=\&paginacao=t\&paginaAtual=3>. Acesso em: 23 dez. 2018.

ARAÚJO NETO, S. E.; FERREIRA, R. L. F.; PONTES, F. S. T. Rentabilidade da produção orgânica de cultivares de alface com diferentes preparos do solo e ambiente de cultivo. Ciência Rural, Santa Maria, v. 39, n. 5, p. 1362-1368, 2009. Disponível em: <https://www.redalyc.org/articulo. oa?id=33113643009>. Acesso em: 23 dez. 2018.

BLAT, S. F.; SANCHEZ, S. V.; ARAÚJO, J. A. C.; BOLONHEZI, D. Desempenho de cultivares de alface crespa em dois ambientes de cultivo em sistema hidropônico. Horticultura Brasileira, Brasília, v. 29, n. 1, p. 135-138, 2011. Disponível em: <https://www.researchgate.net/publication/266839495_ Desempenho_de_cultivares_de_alface_crespa_em_dois_ambientes_de_cultivo_em_sistema_hidroponico >. Acesso em: 23 dez. 2018.

BRAGAGNOLO, N.; MIELNICZUK, J. Cobertura do solo por palha de trigo e seu relacionamento com a temperatura e umidade do solo. Revista Brasileira de Ciência do Solo, Viçosa, v. 14, n. 3, p. 369-374, 1990. Disponível em: <https://www.scienceopen.com/document\%3Fvid\%3Dd298b14e-cfc4-4836-b95d-b84d0c80a9d0+\&cd=2\&hl=pt-BR\&ct=clnk\&gl=br> . Acesso em: 23 dez. 2018.

BRANCO, R. B. F.; SANTOS, L. G. C.; GOTO, R.; ISHIMURA, I.; SCHLICKMANN, S.; CHIARATI, C. S. Cultivo orgânico sequencial de hortaliças com dois sistemas de irrigação e duas coberturas de solo. Horticultura Brasileira, Brasília, v. 28, n. 1, p. 75-80, 2010. Disponível em: <http://www.scielo.br/ scielo.php?script=sci_abstract\&pid=S0102-05362010000100014\&lng=en\&nrm =iso\&tlng=pt $>$. Acesso em: 23 dez. 2018. 
BRANDÃO, A. A.; RIBEIRO, E. C.; FIORINI, C. V. A.; MACHADO, A. F. L.; LOPES, H. M. Dinâmica populacional de plantas daninhas em cultivares de alface produzidas no verão em Seropédica (RJ). Revista Agrogeoambiental, Pouso Alegre, v. 8, n. 1, p. 67-77, 2016. Disponível em: <https://agrogeoambiental.ifsuldeminas.edu.br/index.php/Agrogeoambiental/article/view/782>. Acesso em: 23 dez. 2018.

BURÉS, S. SuBstratos. Madri: Ediciones Agrotécnicas, 1997.341 p. Disponívelem: <http://www.scielo. br/scielo.php?script $=$ sci_nlinks\&ref $=000100 \&$ pid $=S 0102=0536201200010001300004-\&$ Ingen >. Acesso em: 23 dez. 2018.

CARRIJO, O. A.; VIDAL, M. C.; REIS, N. V. B.; SOUZA, R. B.; MAKISHIMA, N. Produtividade do tomateiro em diferentes substratos e modelos de casas de vegetação. Horticultura Brasileira, Brasília, v. 22, n. 1, p. 5-9, 2004. Disponível em: <http://www.scielo.br/scielo.php?script=sci_arttext\&pi$d=$ S0102-0536200400010000 > . Acesso em: 23 dez. 2018.

CARTER, I.; JOHNSON, C. Influence of different types of mulches on eggplant production. Hortscience, Alexandria, v. 23, n. 1, p. 143-145, 1988. Disponível em: <agris.fao.org/agris-search/search.do?recordID $=$ US882680788 > . Acesso em: 23 dez. 2018.

CAVALCANTE, A. S. S. Produção orgânica de alface em diferentes épocas de plantio, preparo e coberturas de solo no Estado do Acre. 2008. 63f. Dissertação (Mestrado), Universidade Federal do Acre, Rio Branco, 2008. Disponível em: <www.seer.ufu.br/index.php/biosciencejournal/article/download/21864/14747> . Acesso em: 23 dez. 2018.

CARVALHO, J. E.; ZANELLA, F.; MOTA, J. H.; LIMA, A. L. S. Cobertura morta do solo no cultivo de alface cv. Regina 2000, em Ji-Paraná, RO. Ciência e Agrotecnologia, v. 29, n. 5, p. 935-939, 2005. Disponível em: <http://www.scielo.br/scielo.php?pid=S1413-70542005000500003\&script=sci_ abstract\&tlng=pt>. Acesso em: 23 dez. 2018.

DALRI, A. B.; CARVALHO NETO, O. F.; MAZZONETTO, F.; CORBANI, R. Z. Fertirrigação com vinhaça concentrada no desenvolvimento da alface. Revista Agrogeoambiental, Pouso Alegre, v. 6, n. 2, p. 93-100, 2014. Disponível em: <https://agrogeoambiental.ifsuldeminas.edu.br/index.php/Agrogeoambiental/article/view/559>. Acesso em: 23 dez. 2018.

FERREIRA, D. F.; CARGNELUTTI FILHO, A.; DAL'COL LÚCIO, A. Procedimentos estatísticos em planejamentos experimentais com restrições na casualização. Boletim Informativo da Sociedade Brasileira de Ciência do Solo, Viçosa, v. 37, n. 1, p. 16-19, 2012. Disponível em: < https://www. researchgate.net/publication/288665818_Procedimentos_estatisticos_em_planejamentos_experimentais_com_restricao_na_casualizacao >. Acesso em: 23 dez. 2018.

FERREIRA, R. L. F.; ALVES, A. S. S. C.; ARAUJO NETO, S. E.; KUSDRA, J. F.; REZENDE, M. I. F. L. Produção orgânica de alface em diferentes épocas de cultivo e sistemas de preparo e coberturas de solo. Bioscience Journal, Uberlândia, v. 30, n. 4, p. 10-17, 2014. Disponível em: < http://www. seer.ufu.br/index.php/biosciencejournal/article/view/21864 >. Acesso em: 23 dez. 2018.

FIALHO, J. F.; BORGES, N. F.; BARROS, N. F. Cobertura vegetal e as características químicas e físicas e atividade da microbiótica de um Latossolo Vermelho-Amarelo Distrófico. Revista Brasileira de Ciência do Solo, Viçosa, v. 15, n. 1, p. 21-28, 1991. Disponível em: < http://www.scielo.br/ 
scielo. php?script =sci_nlinks\&ref=000075\&pid $=$ S0100-6762200300010000500010\&lng $=$ en >. Acesso em: 23 dez. 2018.

FILGUEIRA, F. A. R. Novo manual de olericultura: agrotecnologia moderna na produção e comercialização de hortaliças. 3. ed. Viçosa: UFV, 2013. 421 p. Disponível em: <http://www.bdpa.cnptia. embrapa.br/consulta/busca?b=ad\&id=995452\&biblioteca=vazio\&busca = assunto:Cultivo\&qFacet$\mathrm{s}=$ assunto:Cultivo\&sort=ano-publicacao\&paginacao=t\&paginaAtual=8>. Acesso em: 23 dez. 2018.

GALVÃO, R. O.; ARAÚJO NETO, S. E.; FERREIRA, R. L. F. Plantio direto orgânico de alface sobre cobertura viva e morta e adubada com composto. Agropecuária Científica no Semi-Árido, Campina Grande, v. 9, n. 3, p. 75-80, 2013. Disponível em: <http://revistas.ufcg.edu.br/acsa/index.php/ ACSA/article/view/354>. Acesso em: 23 dez. 2018.

GRANGEIRO, L. C.; COSTA, K. R.; MEDEIROS, M. A.; SALVIANO, A. M.; NEGREIROS, M. Z.; BEZERRA NETO, F.; OLIVEIRA, S. L. Acúmulo de nutrientes por três cultivares de alface cultivadas em condições do Semi-Árido. Horticultura Brasileira, Brasília, v. 24, n. 2, p. 190-194, 2006. Disponível em: < http://www.scielo.br/scielo.php?script=sci_arttext\&pid=S0102-05362006000200013 $>$. Acesso em: 23 dez. 2018.

MÓGOR, A. F.; CÂMARA, F. L. A. Produção de alface no sistema orgânico em sucessão a aveia preta, sobre a palha, e diferentes coberturas do solo. Scientia Agraria, Curitiba, v. 8, n. 3, p. 239-245, 2007. Disponível em: < https://revistas.ufpr.br/agraria/article/view/9535 >. Acesso em: 23 dez. 2018.

MOURA FILHO, E. R. Efeito da cobertura do solo na produtividade da alface. Revista Brasileira de Agrotecnologia, Ipanguaçu, v. 4, n. 2, p. 360-365, 2009. Disponível em: < http://webcache. googleusercontent.com/search?q=cache:tK2P_fQm6GEJ:www.diadecampo.com.br/arquivos/materias/\%257B5778A027-99C9-4B27-8398-74AFD775902C\%257D_2290.pdf +\&cd =1\&hl=p$\mathrm{t}-\mathrm{BR} \& \mathrm{ct}=\mathrm{clnk \& g}=\mathrm{br}>$. Acesso em: 23 dez. 2018.

NASCIMENTO, E. P. Trajetória da sustentabilidade: do ambiental ao social, do social ao econômico. Estudos Avançados, São Paulo, v. 26, n. 74, p. 51-64, 2012. Disponível em: < http://www.scielo. br/scielo.php? pid=S0103-40142012000100005\&script=sci_abstract\&tIng=pt > . Acesso em: 23 dez. 2018.

OLIVEIRA, E. Q.; SOUZA, R. J.; CRUZ, M. C. M.; MARQUES, V. B.; FRANÇA, A. C. Produtividade de alface e rúcula, em sistema consorciado, sob adubação orgânica e mineral. Horticultura Brasileira, Brasília, v. 28, n. 1, p. 36-40, 2010. Disponível em: <www.scielo.br/pdf/hb/v28n1/a07v28n1. pdf>. Acesso em: 23 dez. 2018.

OLIVEIRA, L. C.; STANGARLIN, J. R.; LANA, M. D. C.; SIMON, D.; ZIMMERMANN, A. Biomassa microbiana em cultivo de alface sob diferentes adubações orgânicas e manejo da adubação verde. Revista Brasileira de Agroecologia, Ipanguaçu, v. 4, n. 1, p.182-185, 2009. Disponível em: < http://www.scielo. br/scielo.php?script =sci_nlinks\&ref=000094\&pid $=S 1415=4366201400090001100016-\&$ Ingen>. Acesso em: 23 dez. 2018. 
PINTO, E. N. F.; SOUTO, J. S, LEONARDO, F. A. P.; BORGES, C. H. A.; BARROSO, R. F.; MEDEIROS, A. C. Crescimento de plântulas de alface (Lactuca sativa L.) em solo oriundo de um povoamento de Luetzelburgia auriculata (Allemão) Ducke. Revista Verde de Agroecologia e Desenvolvimento Sustentável, Pombal, v. 11, n. 2, p. 33-38, 2016. Disponível em: < https:/www.gvaa.com.br/revista/ index.php/RVADS/article/view/4260 >. Acesso em: 23 dez. 2018.

QUeIROGA, R. C. F.; NOGUEIRA, I. C. C.; BeZERRA NetO, F., MOURA, A. R. B.; PEDROSA, J. F. Utilização de diferentes materiais como cobertura morta do solo no cultivo de pimentão. Horticultura Brasileira, Brasília, v. 20, n. 3, p. 416-418, 2002. Disponível em: <http://www.scielo. br/scielo.php?pid=S0102-05362002000300003\&script=sci_abstract\&tIng=pt>. Acesso em: 23 dez. 2018.

R Core Team. R: A Language and Environment for Statistical Computing. Vienna, Austria: the R Foundation for Statistical Computing. 2016.

REGHIN, M. Y.; PURISSIMO, C.; FELTRIM, A. L.; FOLTRAN, M. A. Produção de alface utilizando cobertura do solo e proteção das plantas. Scientia Agraria, Curitiba, v. 3, n. 1-2, p. 69-77, 2002. Disponível em: <https://revistas.ufpr.br/agraria/article/view/1033>. Acesso em: 23 dez. 2018.

RODRIGUES, D. S.; NOMURA, E. S.; GARCIA, V. A. Coberturas de solo afetando a produção de alface em sistema orgânico. Revista Ceres, Lavras, v. 56, n. 3, p. 332-335, 2009. Disponível em: < http:// www.ceres.ufv.br/ojs/index.php/ceres/article/view/3439 >. Acesso em: 23 dez. 2018.

SALA, F. C.; COSTA, C. P. Retrospectiva e tendência da alfacicultura brasileira. Horticultura Brasileira, Brasília, v. 30, n. 1, p. 187-194, 2012. Disponível em: <http://www.scielo.br/scielo.php?script=sci_arttext\&pid=S0102-05362012000200002 >. Acesso em: 23 dez. 2018.

SANTI, A.; CARVAlHO, M. A. C.; CAMPOS, O. R.; SILVA, A. F.; ALMEIDA, J. L.; MONTEIRO, S. Ação de material orgânico sobre a produção e características comerciais de cultivares de alface. Horticultura Brasileira, Brasília, v. 28, n. 1, p. 87-90, 2010. Disponível em: < http://www.scielo.br/pdf/hb/ v28n1/a16v28n1 $+\& c d=1 \& h l=p t-B R \& c t=c l n k \& g l=b r>$. Acesso em: 23 dez. 2018.

SEDIYAMA, G. C.; ANDPRATES, J. E. O microclima: possibilidades de modificação. Informe Agropecuário, Belo Horizonte, v. 12, n. 138, p. 39-47, 1986. Disponível em: <http://www.scielo.br/ scielo.php?script=sci_nlinks\&ref=000092\&pid=S0100-204X200200120001400020\&lng=en $>$. Acesso em: 23 dez. 2018.

Silva, E. M. N. C. P.; FerReiRA, R. L. F.; ARAúJO NETO, S. E.; TAVELLA, L. B.; SOlinO, A. J. S. Qualidade de alface crespa cultivada em sistema orgânico, convencional e hidropônico. Horticultura Brasileira, Brasília, v. 29, n. 2, p. 242-245, 2011. Disponível em: <http://www.scielo.br/scielo. php?script=sci_arttext\&pid=S0102-05362011000200019>. Acesso em: 23 dez. 2018.

SILVA, R. F.; PACHECO, L. P.; SOARES, L. S.; FONSECA, W. L.; OLIVEIRA, J. B. S.; SANTOS, A. S. Growth suppression of sandspur grassby cover crops. Pesquisa Agropecuária Tropical, Goiânia, v. 45, n. 3, p. 319-325, 2015. Disponível em: <http://www.scielo.br/scielo.php?script=sci_arttext\&pid=S1983-40632015000300011>. Acesso em: 23 dez. 2018. 
SOUSA, F. M.; PORTELA, G. L. F.; LIMA, M. G.; SOUSA, M. Zoneamento agroclimático da cultura da goiabeira no estado do Piauí, Brasil. Agropecuária Científica no Semi-Árido, Campina Grande, v. 9, n. 3, p. 81-86, 2013. Disponível em: <http://revistas.ufcg.edu.br/acsa/index.php/ACSA/article/ view/257>. Acesso em: 23 dez. 2018.

SOUZA J. L.; RESENDE P. Manual de Horticultura Orgânica. Viçosa: Aprenda Fácil, 2003. 564 p. Disponível em: <https://www.bdpa.cnptia.embrapa.br/consulta/busca\%3Fb\%3Dad\%26id\%3D776054\%26biblioteca\%3DCPAMN\%26busca\%3Dautoria:\%2522RESENDE,\%2520P.\%2522\%26qFacets\%3Dautoria:\%2522RESENDE,\%2520P.\%2522\%26sort\%3D\%26paginacao\%3Dt\%26paginaAtual\%$3 D 1+\& c d=9 \& h l=p t-B R \& c t=c|n k \& g|=b r>$. Acesso em: 23 dez. 2018.

STAL, M. W. A. N. D.; DUSKY, A. J. A. J. Weed control in leafy vegetables: lettuce, endive, escarole and spinach. Disponível em: <http//www edis.ifas.ufl.edu/BODY_WG031>. Acesso em: 23 dez. 2018.

VIANA, T. V. A.; VASCONCELOS, D. V.; AZEVEDO, B. M.; SOUZA, B. F. Estudo da aptidão agroclimática do Estado do Piauí para o cultivo da aceroleira. Ciência Agronômica, Fortaleza, v. 33, n. 2, p. 61-64, 2002. Disponível em: <https://www.bdpa.cnptia.embrapa.br/consulta/busca?b=p$c \& i d=65383 \&$ biblioteca $=$ vazio\&busca $=$ autoria $\% 22$ AZEVEDO,\%20B..$\%$. 20de \%22\&qFacets $=$ autoria:\%22AZEVEDO,\%20B.M.\%20de\%22\&sort=\&paginacao=t\&paginaAtual=1>. Acesso em: 23 dez. 2018.

YURI, J. E.; RESENDE, G. M.; RODRIGUES JÚNIOR, J. C.; MOTA, J. H.; SOUZA, R. J. Efeito de composto orgânico sobre a produção e características comerciais de alface americana. Horticultura Brasileira, Brasília, v. 22, n. 1, p. 127-130, 2004. Disponível em: <https://www.embrapa.br/busca-de-publicacoes/-/publicacao/153995/efeito-de-composto-organico-sobre-a-producao-e-caracteristicas-comerciais-de-alface-americana>. Acesso em: 23 dez. 2018.

Submetido em: 18/08/2016.

Aceito em: 08/11/2017. 
Como citar:

ABNT

MOURA, E. G.; SILVA, F. S.; SILVA, R. F.; ANDRADE, F.R.; SILVA, J. A.; SILVA, A. T. P. Alternative ground cover for lettuce production under tropical climate conditions. Revista Agrogeoambiental, Pouso Alegre, v. 10, n. 4, p. 85-98, dez. Doi: http://dx.doi.org/10.18406/2316-1817v10n420181077

APA

MOURA, E. G., SILVA, F. S., SILVA, R. F., ANDRADE, F. R., SILVA, J. A. \& SILVA, A. T. P. (2018). Alternative ground cover for lettuce production under tropical climate conditions. Revista Agrogeoambiental, 10 (4), 85-98. Doi: http://dx.doi.org/10.18406/2316-1817v10n420181077

ISO

MOURA, E. G.; SILVA, F. S.; SILVA, R. F.; ANDRADE, F. R.; SILVA, J. A. e SILVA, A. T. P. Alternative ground cover for lettuce production under tropical climate conditions. Revista Agrogeoambiental, 2018, vol. 10, n. 4, pp. 85-98. Eissn 2316-1817. Doi: http://dx.doi.org/10.18406/2316-1817v10n420181077

VANCOUVER

Moura EG, Silva FS, Silva RF, Andrade FR, Silva JA, Silva ATP. Alternative ground cover for lettuce production under tropical climate conditions. Rev agrogeoambiental. 2018 dez.; 10(4): 85-98. Doi: http://dx.doi.org/10.18406/2316-1817v10n420181077 